\title{
A wind-tunnel artificially-thickened simulated weakly unstable atmospheric boundary layer
}

\author{
P. E. Hancock, S. Zhang and P. Hayden. \\ EnFlo Laboratory, Faculty of Engineering and Physical Sciences, \\ University of Surrey. \\ Guildford, Surrey, GU2 $7 X H$
}

\begin{abstract}
A wind-tunnel simulation of an atmospheric boundary layer, artificially thickened as is often used in neutral flow wind-loading studies, has been investigated for weakly unstable stratification, including the effect of an overlying inversion. Rather than using a uniform inlet temperature profile, the inlet profile was adjusted iteratively by using measured downstream profiles. It was found that three cycles are sufficient for there to be no significant further change in profiles of temperature and other quantities. Development to nearly horizontally-homogeneous flow took a longer distance than in the neutral case because the simulated layer was deeper and therefore the length scales larger. Comparisons show first-order and second-order moments quantities are substantially larger than given by 'standard forms' in the mixed layer but are close in the surface layer. Modified functions, obtained by matching one to the other, are suggested that amount to an interpolation in the mixed layer between the strongly unstable and the weakly unstable cases.
\end{abstract}

Keywords Atmospheric boundary layer • Convective boundary layer • Scaling laws • Windtunnel experiment

\section{Introduction}

Wind-tunnel experiments on stable and unstable boundary layers mostly employ a boundary layer which has an effective origin at or near the working section inlet, with a fence or trip to promote rapid transition to turbulence. The background to the present work is a study on wind-turbine wake development and the effect of wakes on downwind turbines, in a range of atmospheric stability conditions. This paper is concerned with the setting up of such conditions in the laboratory that meet the requirements of wake studies (such as a suitable size for the model turbines), but not with the wake studies themselves, which will be presented elsewhere. The present results are of interest in themselves. 
If $h$ is the height of the boundary layer under neutral conditions, say, and $H$ is the hub height of a wind turbine, then $H / h$ is a scale of the wind-turbine flow. If $h$ grows in a spatially evolving simulation at a rate ${ }^{1} d h / d x$, then the development length $X$ is $h /(d h / d x)$, and so $X / H=(h / H) /(d h / d x)$. Supposing, at full scale, $h$ $=1 \mathrm{~km}, H=90 \mathrm{~m}$ and $d h / d x=0.02$, leads to $X / H \approx 560$. If, at wind-tunnel scale, $H=0.3 \mathrm{~m}$, then $X \approx 170 \mathrm{~m}$, a length that is impractically long. For a related discussion, see Armit and Counihan (1968). Under stable or unstable conditions the growth rate is respectively smaller or larger, and the observed height of the atmospheric boundary layer is also smaller or larger. Thus, in order to preserve the scale ratio $H / h$ it is necessary from a practical point of view (in order to match the size of model turbine required) to artificially thicken the boundary layer by means of flow generators, and such that the profiles of mean velocity and Reynolds stresses, and also temperature and heat flux, are typical of air flow under various prevailing conditions. Very nearly all work using artificially developed flow has been undertaken in neutral flow, using the classical ellipsoid and fence generators of Counihan (1969) or, for example, the spires and fence of Irwin (1981) that have the advantage of being easier to construct. In practice, strictly maintaining $H / h$ can demand a high working section, and in our work here, in optimizing the scale of flow and the model wind turbines, we have not in fact simulated the whole depth of the simulated atmospheric boundary layer. At a distance $1 \mathrm{~m}$ from the working section floor, the full-scale height is $300 \mathrm{~m}$, which is roughly half the typical height of a neutral boundary layer.

Of particular interest here is that the flow should be slowly evolving over the distance of interest, that is over a distance occupied by a number of turbines in line, and thereby approximately horizontally homogeneous, and consistent in terms of flow statistics with that generally established from meteorological boundary-layer studies. For discussion of aspects of spatial and temporal evolution see, for example, the introduction of Fedorovich et al. (2001).

The present paper covers a series of measurements for the simulation of an unstable boundary layer. The starting point is a previously established simulation

\footnotetext{
${ }^{1}$ Assumed here for convenience to be linear
} 
of a neutral boundary layer for the low roughness of sea-surface conditions, based on ESDU (2001, 2002). The unstable conditions were set up by iteratively adjusting the inlet temperature profile (where the details are given in the following Section). Firstly, for case U1 the floor of the wind tunnel was heated to a temperature above that of the working section inlet air that was also at a uniform temperature. This resulted in profiles of various quantities being free of the initial flow development from about $14 \mathrm{~m}$ onwards from the inlet, with very near similarity in profiles of temperature and other quantities. This naturally led to the question as to whether a more rapid development could be achieved if the flow at the working section inlet, rather than having a uniform temperature profile, had a temperature profile that was equal (in shape and therefore gradients) to that in the downstream flow of this first unstable case. Therefore, a second unstable case, $\mathrm{U} 2$, was generated with a prescribed temperature profile at the working section inlet. This second unstable case then led again to temperature profiles in the downstream part of the flow (from a distance of $14 \mathrm{~m}$ ) that were near similar in shape but different from those of the first unstable case, U1. This second set was then used to generate an inlet profile for a third case, U3, where it was found that the downstream profiles of mean flow and turbulence quantities were very similar in detail to those for U2. In other words, an equilibrium had been established.

Two other cases, U4 and U5, were also investigated in which an inversion was imposed on the top part of the inlet flow. Below the inversion, the inlet profile shape for U4 was identical to that of $\mathrm{U} 2$, and that for U5 was identical to that for U3. For brevity, it is not necessary to give details of all the cases; only those of $\mathrm{U} 1$ and $\mathrm{U} 3$ are given (along with the baseline neutral case, N), and case U5 to show the effect of the imposed inversion. Fuller details are given in Hancock, et al. (2013).

Finally, in addition to addressing the considerations just outlined, further conclusions are drawn from comparisons made with 'standard forms' from the literature for second-order moments of velocity and temperature fluctuations. The poor agreement in the mixed layer is attributed to the weak instability of the present case, the standard forms having been determined for strongly unstable 
flows. Modified forms are given that amount to an interpolation with those for strong instability.

\section{Scaling wind-tunnel measurements}

In the surface layer length and temperature scales are, from Monin-Obukhov similarity,

$$
L_{0}=-\frac{1}{\kappa} \frac{\Theta}{g} \frac{u_{*}^{3}}{(\overline{w \theta})_{0}}
$$

and

$$
\theta_{*}=\frac{-(\overline{w \theta})_{0}}{u_{*}}
$$

respectively, where $u_{*}$ is the friction velocity, $w$ is the fluctuating vertical velocity, $\theta$ is the fluctuating temperature, $(\overline{w \theta})_{0}$ denotes the kinematic surface heat flux, $\Theta$ is the absolute temperature, $\kappa$ is the von Karman constant, and $g$ is the acceleration due to gravity. In the mixed layer, the length scale is taken as the boundary-layer depth, which is usually taken to the bottom of a capping inversion, and the velocity and temperature scales, respectively $w_{*}$ and $\tilde{\theta}_{*}$, are defined as

$$
w_{*}=\left[\frac{g}{\Theta}(\overline{w \theta})_{0} h\right]^{1 / 3},
$$

and

$$
\tilde{\theta}_{*}=\frac{(\bar{w} \boldsymbol{\theta})_{0}}{w_{*}}
$$

(see, for instance, Kaimal and Finnigan, 1994). From Eqs. 1 and 3 we have

$$
\frac{w_{*}}{u_{*}}=\left[\frac{1}{\kappa} \frac{h}{\left|L_{0}\right|}\right]^{1 / 3}
$$

whereupon we see that, if $h /\left|L_{0}\right|$ is to be independent of physical scale, $w_{*} / u_{*}$ is also independent of physical scale. We can write Eq. 3 as 


$$
\left[\frac{w_{*}}{u_{*}}\right]^{3}=\frac{g}{\Theta} \frac{(\overline{w \theta})_{0} h}{u_{*}^{3}} .
$$

If we now suppose the temperature fluctuations scale on a mean temperature gradient and a length scale then $(\overline{w \theta})_{0} \sim w^{\prime} \frac{\partial \Theta}{\partial z} D$ where $w^{\prime}$ denotes the r.m.s. of the vertical velocity fluctuation, $w, D$ is the length scale (such as the wind-turbine rotor diameter) and $z$ is the height above the surface. Thus we have

$$
\left[\frac{w_{*}}{u_{*}}\right]^{3} \sim \frac{g}{\Theta}\left(\frac{w^{\prime}}{u_{*}}\right) \frac{D h}{u_{*}^{2}} \frac{\partial \Theta}{\partial z} .
$$

For similarity the left-hand side and the first part of the right-hand side must each be constant, independent of $D$. Thus, the second part of the right-hand side must also be independent; that is,

$$
\frac{D h}{U_{R}^{2}} \frac{\partial \Theta}{\partial z}=\text { constant }
$$

where we have taken $u_{*}$ as proportional to a reference speed, $U_{R}$. For strict similarity, full-to-model scale $h$ and $D$ must be proportional. $U_{R}$ must of course be taken at geometrically similar positions. This equation shows, in particular, how the temperature gradient must vary with physical scale and reference speed. It implies for the present experiments that the temperature gradient is roughly 2000 times larger than at full scale. (For the wake experiments, $D$ was $416 \mathrm{~mm}$.)

\section{Surface-layer and mixed-layer functional forms}

It is useful to quote at this point 'standard' relationships for mean velocity, mean potential temperature and second-order moments, as functions of height, $z$, and other quantities; as functions of $z / L_{0}$ in the surface layer, and as functions of $z / h$ in the mixed layer. These are based on relationships given by Nieuwstadt and van Dop (1982), Stull (1988) and Kaimal and Finnigan (1994). As yet, there are no universally established functions. Kaimal and Finnigan (1994) give the mixed 
layer as existing between $z \approx 0.1 h$ and $h$, the first of these being typically the height beneath which surface-layer scaling applies in a zero-pressure gradient isothermal boundary layer. See also, for example, Holtslag and Nieuwstadt (1986), Wyngaard (1992). The relationships cited below have been obtained almost entirely from measurements in the strongly unstable boundary layer, where $L_{0}<h$ by an order of magnitude or more. See, for instance, Kaimal et al. (1976). This condition does not apply here, where the stratification is weakly unstable. We take the surface layer as existing beneath $z \approx 0.1 \mathrm{~h}$. On this basis, a matching layer (Kaimal and Finnigan, 1994) between the surface layer and $0.1 h$ would appear not to exist.

\subsection{Surface layer}

Assuming for the unstable surface layer that the mean velocity, $U$, and mean temperature are given by

$$
\frac{\partial U}{\partial z}\left(\frac{\kappa z}{u_{*}}\right)=\phi_{m}
$$

and

$$
\frac{\partial \Theta}{\partial z}\left(\frac{\kappa z}{\theta_{*}}\right)=\phi_{h}
$$

where $\phi_{m}=\left[1+16 z /\left|L_{0}\right|\right]^{-1 / 4}$ and $\phi_{h}=\left[1+16 z /\left|L_{0}\right|\right]^{-1 / 2}$, and then the following wellestablished $^{2}$ forms for $U$ and $\Theta$ are obtained.

$$
\frac{U}{u_{*}}=\frac{1}{\kappa}\left[\ln \left(\frac{z}{z_{0}}\right)-\psi\left(\frac{z}{\left|L_{0}\right|}\right)+\psi\left(\frac{z_{0}}{\left|L_{0}\right|}\right)\right]
$$

where $\psi\left(\frac{z}{\left|L_{0}\right|}\right)-\psi\left(\frac{z_{0}}{\left|L_{0}\right|}\right)=\ln \left(\frac{(1+x)^{2}}{\left(1+x_{0}\right)^{2}} \frac{\left(1+x^{2}\right)}{\left(1+x_{0}{ }^{2}\right)}\right)-2\left(\tan ^{-1}(x)-\tan ^{-1}\left(x_{0}\right)\right)$ and $x$ and $x_{0}$ are given by $x=\left[1+16 z /\left|L_{0}\right|\right]^{+1 / 4}$ and $x_{0}=\left[1+16 z_{0} /\left|L_{0}\right|\right]^{+1 / 4}$. And,

\footnotetext{
${ }^{2}$ but repeated here for convenience. See, for instance, Stull (1988).
} 


$$
\frac{\Theta-\Theta_{0}}{\theta_{*}}=\frac{1}{\kappa}\left[\ln \left(\frac{y-1}{y+1}\right)-\ln \left(\frac{y_{0}-1}{y_{0}+1}\right)\right]
$$

where $y=\left[1+16 z /\left|L_{0}\right|\right]^{+1 / 2}, \quad y_{0}=\left[1+16 z_{0 \theta} /\left|L_{0}\right|\right]^{+1 / 2}$ and $\Theta_{0}$ is the surface temperature. Here, $z_{0}$ and $z_{0 \theta}$ are the roughness lengths for velocity and temperature. For the (kinematic) Reynolds stresses $\overline{u^{2}}, \overline{v^{2}}$ and $\overline{w^{2}}$, Kiamal and Finnigan (1994) give

$$
\frac{\overline{u^{2}}}{u_{*}^{2}}=\frac{\overline{v^{2}}}{u_{*}^{2}}=4+0.6\left(\frac{h}{\left|L_{0}\right|}\right)^{2 / 3},
$$

and

$$
\frac{\overline{w^{2}}}{u_{*}^{2}}=1.25^{2}\left(1+3 \frac{z}{\left|L_{0}\right|}\right)^{2 / 3},
$$

and for the mean-square of the temperature fluctuation, $\overline{\theta^{2}}$,

$$
\frac{\overline{\theta^{2}}}{\theta_{*}^{2}}=4\left(1+9.5 \frac{z}{\left|L_{0}\right|}\right)^{-2 / 3},
$$

where $u$ and $v$ are, respectively, the streamwise and transverse velocity fluctuations.

\subsection{Mixed layer}

Nieuwstadt and van Dop (1982) quoting Lenschow and Stephens (1980) give

$$
\frac{\overline{u^{2}}}{w_{*}^{2}}=\frac{\overline{v^{2}}}{w_{*}^{2}} \approx 0.4
$$

while Kiamal and Finnigan (1994) quoting Caughey and Palmer (1979) and Adrian et al. (1986) suggest a constant of 0.35. They also quote Hunt et al. (1988) for $\overline{w^{2}}$ :

$$
\frac{\overline{w^{2}}}{w_{*}^{2}}=1.8\left(\frac{z}{h}\right)^{2 / 3}\left(1-0.8 \frac{z}{h}\right)^{2}
$$

and Lenschow et al. (1980) and Kaimal et al. (1976) for $\overline{\theta^{2}}$, 


$$
\frac{\overline{\theta^{2}}}{\tilde{\theta}_{*}^{2}}=1.8\left(\frac{z}{h}\right)^{-2 / 3}
$$

in the range $0.1 h<z<0.5 h$. The first parts of Eq. 17 and Eq. 18 are in fact consistent with matching-layer scaling in which neither the surface shear stress nor boundary-layer height are parameters.

\section{Wind tunnel, instrumentation and reference neutral boundary layer}

\subsection{Wind tunnel and instrumentation}

The measurements were made in the EnFlo wind tunnel that has a working section $20 \mathrm{~m}$ long, $3.5 \mathrm{~m}$ wide and $1.5 \mathrm{~m}$ high. (Some details are also given in Robins et al. 2001) It is an open-return suck-down type with 15 heaters at the working section inlet (each covering $100 \mathrm{~mm}$ in height) and a heat exchanger between the end of the working section and the fans, supplied by a chilled water system. As only a $3 \mathrm{~m}$ width of the floor is heated or cooled, Perspex side panels were employed in all cases, from the working section inlet to $X=18 \mathrm{~m}$, where $X$ is the distance from the inlet. The first $7 \mathrm{~m}$ of the floor was heated by means of the original floor panels, while the subsequent $11 \mathrm{~m}$ was heated by 36 heating plates, measuring $0.33 \mathrm{~m} \times 2.95 \mathrm{~m} \times 5 \mathrm{~mm}$ thick. The plates and floor panels were controlled in closed-loop control systems. Allowance for a discrepancy between thermocouples was effected by recording the temperatures in the neutral case, and adjusting the 'demand temperature' accordingly. The Irwin spires were formed from slightly truncated triangles, $1490 \mathrm{~mm}$ high, $150 \mathrm{~mm}$ wide at the bottom, 10 $\mathrm{mm}$ wide at the top, spaced laterally $660 \mathrm{~mm}$. (No fence was employed.) The surface roughness elements were sharp-edged brick-like blocks $50 \mathrm{~mm}$ wide, 16 $\mathrm{mm}$ high, $5 \mathrm{~mm}$ thick, standing on the $50 \mathrm{~mm} \times 5 \mathrm{~mm}$ face. They were placed in a staggered arrangement with streamwise and lateral pitches of $360 \mathrm{~mm}$ and 510 $\mathrm{mm}$, respectively, with alternate rows displaced laterally to give the staggered pattern. 
The laser Doppler anemometer (LDA) probe, cold-wire probe and a thermocouple probe were supported on the main wind-tunnel traverse, and moveable between $z$ $=40 \mathrm{~mm}$ and $1050 \mathrm{~mm}$. A lower limit on $z$ risked the LDA probe hitting a roughness element. Moreover, measurements at a lower height would have been influenced more by the local flow generated by the individual roughness elements, rather than the smoothed-out effect seen at larger $z$. The cold wire was set at about $3 \mathrm{~mm}$ behind the LDA measuring volume, and the thermocouple set to the side separated by about $15 \mathrm{~mm}$ from the measuring volume. The measuring volume of the Dantec $27 \mathrm{~mm}$ FibreFlow probe was about $3 \mathrm{~mm}$ in length, and so an offset of the cold wire by this distance was deemed satisfactory. In some earlier measurements the cold wire had been much closer, about $1 \mathrm{~mm}$ from the measuring volume, and it was found this accounted for some errors in the LDA measurements, including spuriously large vertical mean velocity, $W$, and a small positive $\overline{u w}$ at the top of the layer. While the probe disturbance field would not have given the observed error in $W$ it was supposed the error was caused by relatively strong backscatter from the probe wire or prongs of a weak light level beyond the nominal edge of the beam. A separation of $2 \mathrm{~mm}$ could have been used but a margin of safety was preferred with a separation of $3 \mathrm{~mm}$. The cold wire probe was calibrated against the mean temperature of the thermocouple. A second thermocouple was set at $0.45 \mathrm{~m}$ above the first, so that the mean temperature profile could be measured over the full height of the working section. Only the streamwise and vertical velocity components were measured. The surface temperature for cases U2-U5 was determined by reference to a temperature at the inlet at a height of $z=550 \mathrm{~mm}$; this will be discussed in more detail in Sect. 3.1.

Now, while conditions are sought, downstream of the development zone of the working section, that are slowly varying with $X$, there cannot be strict thermal equilibrium. Regarding the latter part of the working section as a control volume, there are advected heat fluxes at inlet and exit, and a surface heat flux at the floor. There was negligible heat flux through the roof, however. A heat flux at the roof equal in magnitude to that at the floor would give equal advected heat fluxes. An approximate calculation for the present measurements (case U3 in particular) 
shows that the profiles in the downstream part of the working section should have temperatures about $0.3{ }^{\circ} \mathrm{C}$ higher than in the inlet profiles.

A reference ultrasonic anemometer was placed at $X=5 \mathrm{~m}, z=1 \mathrm{~m}, y=-0.78 \mathrm{~m}$, where $y$ is the lateral position from the centreline. The reference speed, $U_{\mathrm{REF}}$, for all the measurements was $1.5 \mathrm{~m} \mathrm{~s}^{-1}$. Previous measurements had shown the neutral case to be Reynolds-number independent, and no further checks were made in this regard as it was expected that roughness effects as such would not be influenced by unstable (or stable) stratification since the associated length scales are much smaller than that of the energy-containing motions of the flow as a whole. Indeed, testing for Reynolds number independence is more complicated in a stratified flow because the temperature field would also have to be adjusted for change in flow speed.

Sample periods for each point were 3 mins; ideally, this would have been longer, but the primary objective in the first instance was to observe trends as a result of changing the inlet temperature profile. The main source of error in first-order and second-order moments was in the statistical sampling interval, for which the error bands are about $\pm 1 \%$ for mean velocity and about $\pm 10 \%$ for Reynolds stresses and turbulent heat flux. Based on the scatter within sets of profiles a sample time of about twice this duration would have been preferable. Non-dimensional parameters such as the correlation coefficients $\overline{u w} / u^{\prime} w^{\prime}$ and $\overline{w \theta} / w^{\prime} \theta^{\prime}$ are much smoother, where $u^{\prime}=\left(\overline{u^{2}}\right)^{1 / 2}$ and $\theta^{\prime}=\left(\overline{\theta^{2}}\right)^{1 / 2}$. The absolute uncertainty in $z$ was about $\pm 2 \mathrm{~mm}$ owing to a slight undulation in the floor and variation in the probe traverse rails system, but the error in the intervals in $z$ was negligible. Data acquisition was by means of the standard LabView based software system of the laboratory.

\subsection{Baseline neutral boundary layer}

Although details of the reference boundary layer are also given later, it is helpful to give first the basis of the neutral case. The basis is the ESDU $(2001,2002)$ guidelines, adjusted to a reference speed of $10 \mathrm{~m} \mathrm{~s}^{-1}$ at a height of $10 \mathrm{~m}$, for a 
typical offshore sea-surface roughness, and a full-to-model scale ratio of 300:1. The Irwin-like spires and roughness elements were adjusted iteratively in shape, size and spacing in order to match the expected form of mean velocity profile (given later) and the turbulence intensities $u^{\prime} / U, v^{\prime} / U$ and $w^{\prime} / U$, where $v^{\prime}=\left(\overline{v^{2}}\right)^{1 / 2}$. In this investigation only $u^{\prime}$ and $w^{\prime}$ were measured. The earlier measurements had shown that the vertical fluctuation was the slowest to settle to near-constant conditions in the outer part of the boundary layer, and that $w^{\prime}$ adjusts more slowly than $u^{\prime}$ can be seen in Fig. 1. Near the surface, where the energy-containing length scales are smaller, the flow settles more rapidly. The broken and full lines in this figure are for a surface roughness at full scale of $0.005 \mathrm{~m}$ and $0.0005 \mathrm{~m}$ (see also Stull, 1988). Magnusson and Smedman (1996), for example, took $z_{0}=0.0005 \mathrm{~m}$ in their offshore field study; $u_{*}$ and $z_{0}$ are given in Table 2. It should be remembered that, for low surface roughness such as that over a sea surface, as opposed to, say, urban roughness, the roughness length in a wind tunnel is not in proportion to that at full scale (by the geometric scale ratio); the roughness has to be disproportionately larger in order to maintain Reynolds number independence. This last point was investigated by Hancock and Pascheke (2013), where other details can be found.

\section{$5 \quad$ Results}

\subsection{Mean temperature profiles}

Before presenting the mean velocity and Reynolds stress profiles for each case, the cases are defined first in terms of their respective mean temperature profiles. Figs 2a-c show the profiles at the working-section inlet and at downstream stations, for U1, U3 and U5, respectively. Fig. 2b shows in particular that the shape of the downstream profiles is very close to that at the inlet, in contrast to that seen in Fig. 2a. Overall, the results in each unstable case exhibited near invariance from about $X=14 \mathrm{~m}$ onwards. For clarity, although measurements were made at $1 \mathrm{~m}$ intervals from $X=6 \mathrm{~m}$, only those from later stations are shown. 
Table 1 gives examples of the surface working temperature, $T_{s}$, an inlet profile reference temperature, $T_{\text {Iref }}$, at a height of $550 \mathrm{~mm}$, and the difference, nominally $18 \mathrm{~K}$. When necessary, the surface temperature, $T_{s}$, was adjusted so as to always give the differences given in Table 1 in order to allow for a higher laboratory-air inlet temperature in a particular run, as the inlet heaters could only increase temperature, obviously.

Table 1 Surface and inlet reference temperatures for the three cases.

\begin{tabular}{lccc}
\hline Case & $T_{s}\left({ }^{\circ} \mathrm{C}\right)$ & $T_{\text {Iref }}\left({ }^{\circ} \mathrm{C}\right)$ & Diff $\left({ }^{\circ} \mathrm{C}\right)$ \\
\hline U1 & 43.0 & 24.5 & 18.5 \\
U3 & 45.0 & 27.3 & 17.7 \\
U5 & 45.0 & 27.3 & 17.7 \\
\hline
\end{tabular}

For the surface-layer profile (Eq. 12) of Fig. 2, $\Theta_{0}$ has been taken as the surface temperature, $T_{s}$, but adjusted by a relatively small amount (i.e. between 0 and 0.7 ${ }^{\circ} \mathrm{C}$ ) in order to match the respective measured profiles, as given in Table 2. The temperature profile roughness length $z_{0_{\theta}}$ has been taken as constant at $0.002 \mathrm{~mm}$, as also given in Table 2. A smaller value for $z_{0_{\theta}}$ would lower the $\Theta_{0}$ required for a fit with the measurements, but finer adjustment is not warranted in the present context or measurement precision. For a discussion about the roughness length for temperature see, for example, Beljaars and Holtslag (1991).

For case U3 it will be noticed that the downstream profiles are all at a higher temperature than at the inlet, by about $1{ }^{\circ} \mathrm{C}$. A higher temperature is to be expected as there was negligible heat transfer through the roof of the wind tunnel, but only by about $0.3{ }^{\circ} \mathrm{C}$, as explained earlier. The remaining difference in temperature is attributed to differences between thermocouples. Though not shown in Fig. 2, the profiles from $X=6 \mathrm{~m}$ for case U1 show a progressive internal-layer-like development. Any such equivalent adjustment is much more difficult to see for cases $\mathrm{U} 3$ or U5, because the inlet profile is much closer to the downstream profiles. 
In case U5, the inversion gradient is much smaller than it is at the inlet, in contrast to the unstable part of the profile, which differs relatively little. It is assumed the smaller gradient in the inversion region is because of mixing imposed from below. At the downstream stations the gradient is roughly $3 \mathrm{~K} \mathrm{~m}^{-1}$. From Eq. 8, this amounts to a full-scale gradient of roughly $+0.002 \mathrm{~K} \mathrm{~m}^{-1}$, which is not untypical.

As in the figures considered so far, all quantities are given at wind tunnel scale. Reference to boundary layer depth, $h$, is deferred except in a few instances until Sect. 6. $h$ was in the range 1100 to $1400 \mathrm{~mm}$.

\subsection{Mean velocity profiles}

Fig. 3 shows the mean velocity profiles for the four cases, together with the surface-layer Eq. 11. Velocity, length and temperature scales are as given in Table 2. A marked feature in each of the unstable cases is the much fuller velocity profile and the sharp 'knee' at $z \approx 100 \mathrm{~mm}$ which, as will be seen, is roughly at $0.1 \mathrm{~h}$. Above the knee, there is clearly a fairly constant gradient out to the furthest measurement point. The $u_{*}$ given in Table 2 is based on the shear stress profiles (Fig. 6). It is very nearly the same for each unstable case. The roughness length is slightly smaller for the neutral case to give best fit to that set of mean velocity profiles, although it was expected it would be the same for all cases. As the difference is small and does not affect any conclusions drawn here it is not considered further. 
Table 2 Scale parameter values for each of the four cases.

\begin{tabular}{lcccc}
\hline & $\mathrm{N}$ & $\mathrm{U} 1$ & $\mathrm{U} 3$ & $\mathrm{U} 5$ \\
\hline$U_{\mathrm{REF}}\left(\mathrm{m} \mathrm{s}^{-1}\right)$ & 1.5 & 1.5 & 1.5 & 1.5 \\
$u_{*} / U_{\mathrm{REF}}$ & 0.045 & 0.055 & 0.055 & 0.055 \\
$z_{0}(\mathrm{~mm})$ & 0.09 & 0.10 & 0.10 & 0.10 \\
$z_{0 \theta}(\mathrm{mm})$ & - & 0.002 & 0.002 & 0.002 \\
$\theta_{*}\left({ }^{\circ} \mathrm{C}\right)$ & - & -0.61 & -0.57 & -0.57 \\
$\Theta_{0}\left({ }^{\circ} \mathrm{C}\right)$ & - & 43.0 & 45 & 45.0 \\
$(\overline{w \theta})_{0}\left(\mathrm{~K} \mathrm{~m} \mathrm{~s}^{-1}\right)$ & - & 0.05 & 0.047 & 0.047 \\
$(\overline{w \theta})_{1}\left(\mathrm{~K} \mathrm{~m} \mathrm{~s}^{-1}\right)$ & - & 0.062 & 0.062 & 0.062 \\
$\left|L_{0}\right|(\mathrm{mm})$ & - & 893 & 956 & 956 \\
$h(\mathrm{~mm})$ & - & 1100 & 1400 & 1200 \\
\hline
\end{tabular}

\subsection{Reynolds stresses}

Profiles of Reynolds stress $(\times-1)$ are shown in Figs 4 to 6 . (No measurements were made of $\overline{v^{2}}$.) When compared with the neutral case all the stresses are much larger. $\overline{w^{2}}$ is the most distinctive in that it shows a steep increase with $z$, rather than a nearly monotonic decrease, reaching a level that is in excess of a factor 3 larger in terms of maxima. The shear stress is nearly constant beneath $z$ of about $600 \mathrm{~mm}$. The shapes of the profiles of $\overline{u^{2}}$ and $\overline{w^{2}}$ for cases U3 and U5 are comparable with those of $\mathrm{U} 1$, but exhibit a region in which $\overline{u^{2}}$ is nearly flat, and a peak in $\overline{w^{2}}$ that is more rounded, larger and further from the surface, and more like features seen in the field data (see e.g. Kaimal and Finnigan, 1994). The profiles of $\overline{u w}$ for U3 and U5 each show a small rise in magnitude with $z$ near the surface, while any such trend for case U1 is much less marked. It is assumed this difference with respect to case U1 is associated with the higher level of $\overline{w^{2}}$ seen in $\mathrm{U} 3$ and $\mathrm{U} 5$. In all the unstable cases, the variation with increasing $X$ is least near the surface and largest in the upper part of the flow, with $\overline{w^{2}}$ taking the longest distance to adjust, as also observed in the neutral case. That there is more variation with $X$ away from the surface is to be expected because of the larger length scales of the turbulent motions. 
Fig. 7 shows the correlation coefficient $\overline{u w} / u^{\prime} w^{\prime}$. The variation from profile to profile in a set is less than that for the Reynolds stress profiles themselves, (consistent with a shorter than ideal sample time for each measurement point). The shape and level of the profiles for each of the unstable cases are very comparable one with another. Though somewhat larger in magnitude than in the neutral case they differ much less than do the Reynolds stress profiles. That $\overline{u w} / u^{\prime} w^{\prime}$ is larger implies some difference in structure and a more efficient transfer of momentum, as would be anticipated for the contribution from buoyant convection.

\subsection{Turbulent heat flux and temperature fluctuations.}

The vertical heat flux, $\overline{w \theta}$, is shown in Fig. 8 , and the mean square of the temperature fluctuations, $\overline{\theta^{2}}$, in Fig. 9. (The horizontal heat flux $-\overline{u \theta}$ was also measured but is not shown.) Note, neither of these quantities are given in nondimensional terms. For case U1, $\overline{w \theta}$ shows as a trend a slight rise with height near the surface, followed by an approximately constant level as far as about $z \approx$ $400 \mathrm{~mm}$, and then a more or less linear decrease to zero (at $z \approx 1100 \mathrm{~mm}$ ). In the other two cases the rise near the surface is more marked, with the decrease not occurring until $z \approx 700 \mathrm{~mm}$. The higher level of $\overline{w \theta}$ for U3 and U5 is consistent with the higher level of $\overline{w^{2}}$ in these cases. However, another point needs to be made, which is about the rise in $\overline{w \theta}$ over the first $100 \mathrm{~mm}$ or so. In a precisely parallel flow the gradient $\partial \overline{w \theta} / \partial z$ should be zero (above any viscous effects, which are taken as negligible). The present measurements were not made with the purpose of making a detailed investigation of the terms in the mean-temperature transport equation, so only limited comment can be made in this regard. In a growing layer $W$ is small but positive and, as can be seen from Fig. $2, \partial \Theta / \partial z$ is large and negative; it is supposed this may account for the observed rise in $\overline{w \theta}$. Ohya and Uchida (2004), whose measurements are discussed later in Sect. 7, also observed a rise comparable to that here. 
The correlation coefficient, $\bar{w} \boldsymbol{\theta} / w^{\prime} \theta^{\prime}$, given in Fig. 10, shows an almost constant rise with $z$, showing that the larger-scale turbulence further from the surface is more efficient in transporting heat vertically. The correlation coefficient $\overline{u \theta} / u^{\prime} \theta^{\prime}$ (not shown) in contrast shows the opposite trend, and $\overline{\theta^{2}}$ in Fig. 9 shows a monotonic decreases in each case. The profiles of $\overline{w \theta}$ and also $\overline{w \theta} / w^{\prime} \theta^{\prime}$ differ from that generally reported in the literature, where a roughly linear decrease with $z$ is given. See, for instance, Huynh, et al. (1990). Now, interestingly, the measurements of Ohya and Uchida (2004) show the development of roughly constant $\overline{w \theta}$ to a greater height with progressively weaker instability, qualitatively comparable with that here. Their measurements also show roughly constant $\overline{u w}$, again comparable with that here. Their case E1 is very close in terms of $h /\left|L_{0}\right|$, namely $\approx 1.5$, to our measurements. Further discussion is deferred until Sect. 6.

The inversion imposed in case U5 (compared with U3) tends to give more rapid decreases in $\overline{w \theta}$ and $\overline{\theta^{2}}$, and also in $\overline{w^{2}}$, at the boundary layer top.

\subsection{Buoyant production of turbulent kinetic energy.}

The ratio of buoyant production to total production, $\left(1+\frac{-\overline{u w}}{\overline{w \theta}} \frac{\Theta}{g} \frac{\partial U}{\partial z}\right)^{-1}$, is shown in Fig. 11. What is most striking is that while this ratio is 'low' near the surface it reaches about 0.6 in the upper part of the flow. The trends in the profiles indicate, too, a peak between $z=200 \mathrm{~mm}$ and $300 \mathrm{~mm}$. In terms of surface-layer similarity, this ratio is $\left(1+\left|L_{0}\right| / z\right)^{-1}$, which is also shown in Fig. 11. By itself, the surface-layer trend given by this relationship would suggest a much lower level of buoyant production further from the surface, but though the measurements near the surface are close to this trend they rise well above it with increasing height. Thus, although the flow is weakly unstable in terms of $\left|L_{0}\right|$, the buoyant production over much of the layer is large, and accounts for the high level of $\overline{w^{2}}$. As noted earlier, the heat flux correlation coefficient, $\bar{w} \theta / w^{\prime} \theta^{\prime}$, shows an increase in the 'efficiency' of the turbulence in transferring heat with increasing height. 
The energy balance by Caughey and Wyngaard (1979), though only extending to about $0.2 h$, shows a much more rapid rise near the surface, consistent with their much smaller $\left|L_{0}\right|$, the ratio reaching a peak of about 0.9 . That of Lenschow et al. (1980, see also Wyngaard, 1992) reaches 0.98 at $0.4 h$.

\section{Modified functional forms}

The functional forms given in Sect. 3 for $\overline{\theta^{2}}$, namely Eqs 15 and 18, are shown in Fig. 9, but with a modification to the multiplying factor in Eq. 15 for the surface layer:

$$
\frac{\overline{\theta^{2}}}{\theta_{*}^{2}}=5.7\left(1+9.5 \frac{z}{\left|L_{0}\right|}\right)^{-2 / 3}
$$

The adjustment to this factor is discussed further, below. The match in the mixed layer is good for U3 and U5, and good in part for U1. However, this is not the case for quantities involving velocity fluctuations in the mixed layer. Eq. 17 for $\overline{w^{2}}$ can be re-written in terms of $u_{*}$ as

$$
\frac{\overline{w^{2}}}{u_{*}^{2}}=1.8\left(\frac{h}{\kappa\left|L_{0}\right|}\right)^{2 / 3}\left(\frac{z}{h}\right)^{2 / 3}\left(1-0.8 \frac{z}{h}\right)^{2} .
$$

As such it is not consistent in terms of an 'overlap'3 with Eq.14, for all $\left|L_{0}\right| / h$. There is only concurrence when $\left|L_{0}\right| / h$ is $\mathrm{O}(0.01)$, which is not surprising in that the relationships have been derived for strongly unstable flows. For the present measurements, Eq. 20 (or 17) gives a substantially lower $\overline{w^{2}}$, by a factor of about 2.4. Even so, the shape, with a suitably chosen value for $h$ (as discussed below) is very comparable to the measured profiles. By introducing a multiplying factor, $A$, re-writing Eq. 20 as

$$
\frac{\overline{w^{2}}}{u_{*}^{2}}=1.8 \mathrm{~A}\left(\frac{h}{\kappa\left|L_{0}\right|}\right)^{2 / 3}\left(\frac{z}{h}\right)^{2 / 3}\left(1-0.8 \frac{z}{h}\right)^{2},
$$

\footnotetext{
${ }^{3}$ Term used here to mean equal at a point, but not continuity of gradient.
} 
and requiring this equation to match Eq. 14 at some fraction of $h$, we get

$$
\frac{\overline{w^{2}}}{u_{*}^{2}}=8.57\left(1+0.3 \frac{h}{\left|L_{0}\right|}\right)^{2 / 3}\left(\frac{z}{h}\right)^{2 / 3}\left(1-0.8 \frac{z}{h}\right)^{2}
$$

where the match has been made at $z / h=0.1$. But, a comparison would show that Eq. 14 is itself not that good a fit to the measurements near the surface. A better fit is given by

$$
\frac{\overline{w^{2}}}{u_{*}^{2}}=1.1^{2}\left(1+8 \frac{z}{\left|L_{0}\right|}\right)^{2 / 3}
$$

The factor of $1.1^{2}$ is much closer to the value of this ratio in zero-pressuregradient isothermal-boundary layers (e.g. Hancock and Bradshaw, 1989, which also includes the effect of increased external turbulence), and to the present neutral case, N. Combining this last equation with that of Eq. 21 gives

$$
\frac{\overline{w^{2}}}{u_{*}^{2}}=6.63\left(1+0.8 \frac{h}{\left|L_{0}\right|}\right)^{2 / 3}\left(\frac{z}{h}\right)^{2 / 3}\left(1-0.8 \frac{z}{h}\right)^{2}
$$

The curves given in Fig. 5 are according to these last two equations, where in the latter $h$ has been chosen so at to give a 'by-eye' good fit to the measurements. In the case U5 the height so defined lies slightly above the minimum in the mean temperature profiles (Fig. 3c), and at a point at which the vertical heat flux (Fig. 8c) might reasonably be extrapolated to zero. In cases U1 and U3, where there is no imposed inversion the height corresponds to the height at which the temperature gradient has become small.

Other expressions for $\overline{w^{2}}$ that depend upon both surface layer and mixed layer scales have been proposed, such as those by Gryning, et al. (1987) and Holtslag and Moeng (1991), which are superpositions from shear-driven and buoyancydriven contributions. These do not fit well the present measurements. 
A related lack of concurrence exists for $\overline{u^{2}}$ in that Eq. 16 gives a level that is also well below that measured. Yet the measured profiles of this stress as well as that of $\overline{w^{2}}$ are comparable in form to the examples given by Caughey and Palmer (in Nieuwstadt and van Dop, 1982) or Kaimal and Finnigan (1994). Here, it is proposed that $\overline{u^{2}}$ (and $\overline{v^{2}}$ ) should remain in fixed proportion to $\overline{w^{2}}$. From Eq. 17 the maximum in $\overline{w^{2}}$ is $\left.\overline{w^{2}}\right|_{M A X}=0.47 w_{*}^{2}$, and so from Eq. 16 $\left.\overline{u^{2}} \overline{/ w^{2}}\right|_{\text {MAX }}=\overline{v^{2}} /\left.\overline{w^{2}}\right|_{M A X} \approx 0.8$. From the maximum for $\overline{w^{2}}$ given by Eq. 22 it follows that

$$
\frac{\overline{u^{2}}}{u_{*}^{2}}=\frac{\overline{v^{2}}}{u_{*}^{2}}=1.78\left(1+0.3 \frac{h}{\left|L_{0}\right|}\right)^{2 / 3},
$$

or, from Eq. 24, that

$$
\frac{\overline{u^{2}}}{u_{*}^{2}}=\frac{\overline{v^{2}}}{u_{*}^{2}}=1.37\left(1+0.8 \frac{h}{\left|L_{0}\right|}\right)^{2 / 3}
$$

For the present measurements, these two relationships differ by no more than about $2 \%$. Fig. 4 shows $\overline{u^{2}} / U_{\mathrm{REF}}^{2}$ as given by Eq. 13 and by Eq. 26 . The surface layer value is consistent with the trend in the near-surface measurements and the level for the mixed layer is notably close to the measured level, in each case. For U1, though, the measured profiles do not have a flat region, continuing to decrease above about $z / h=0.35$.

Returning to the functional forms for $\overline{\theta^{2}}$ (see Fig. 9), the same approach can be adopted to give a match at, again, say $z / h=0.1$. This leads to a term replacing the 4 in Eq. 15 with $\approx 4.4\left(1+1.0\left|L_{0}\right| / h\right)^{2 / 3}$, which for the present measurements gives a factor of 6.3 rather than 5.7 in Eq. 19.

\section{Comparison with the measurements of Ohya and Uchida (2004)}

While further comparisons could be made using measurements from various sources, Ohya and Uchida (2004) is particularly useful as measurements (at one 
streamwise station) are given for a range of convective instability covering that investigated here. They used only a turbulence trip at the working section entrance, and did not use spires or similar devices to provide artificial thickening. In their weakest stability case (E1) the ratio $h /\left|L_{0}\right|$ is 1.5 , very close to that in our experiments, viz. 1.2 to 1.5 . Fig. 12 shows $\overline{w^{2}}$ and $\overline{u^{2}}$ for cases U3 and U5 against $z / h$, normalized in the same way as Ohya and Uchida (2004) by a modified mixed-layer velocity scale $w_{* * *}$, together with their data. Here, $w_{* *}$ is defined in the same way as $w_{*}$ except that the heat flux is taken as an average maximum in the trend of the profiles, denoted $(\overline{w \theta})_{1}$, rather than as an inferred surface value; $(\overline{w \theta})_{1}$ is also given in Table 2 . The present measurements compare fairly well with Ohya and Uchida (2004), the comparison being better overall for case U5. This figure also shows $\overline{w^{2}}$ from Eq. 24, but scaled with respect to $w_{* * *}$.

Comparisons for $\overline{u^{2}}$ are good above $z / h \approx 0.3$ in case U5, but not so good for case $\mathrm{U} 3$, and their measurements rise to notably higher values nearer the surface. Their near-surface $\overline{u^{2}} / w_{* *}^{2}$ values are larger than the consensus of other data, a point they unfortunately do not comment on. Although their study is for a strong inversion imposed above, while that here is either non-existent or small, there is no obvious reason to suppose this accounts for differences in the near-surface behaviour of $\overline{u^{2}}$. More likely is the fact that they were primarily interested in the mixed and entrainment layer and that the surface was kept smooth (Ohya, private communication, 2012) for the purpose of the direct numerical simulations. Overall, the main point to draw from their measurements is that both $\overline{u^{2}} / w_{* *}^{2}$ and $\overline{w^{2}} / w_{\text {*** }}^{2}$ show very clearly decreases in magnitude with increasing convective instability, by a factor of roughly 3 when $h /\left|L_{0}\right|$ has reached 15 . Given that $u_{*}$ and $w_{*}$ are both scales of the flow, it is not surprising that for weak $w_{*} / u_{*}$ there is significant dependence upon this parameter, while for purely free convection $u_{*}$ is no longer relevant.

Other comparisons with their measurements also show reasonable agreement. Fig. 13 shows profiles of shear stress and vertical heat flux for cases U3 and U5, where $\overline{w \theta}$ is normalized by $w_{* *}$ and $\tilde{\theta}_{* * *}$, where $\tilde{\theta}_{* *}=(\overline{w \theta})_{1} / w_{* * *}$. Our profiles are 
close to theirs in shape, including the rising trend in $\overline{w \theta}$ near the surface. (They used hot-wire anemometry rather than LDA for their velocity measurements.) Their smooth surface and the higher level of $\overline{u^{2}}$ and a higher level of turbulence production, it is assumed, could account for some of the remaining differences seen in the comparisons with the present data.

\section{Concluding comments}

The first of two main conclusions is that in the present wind-tunnel arrangement about $14 \mathrm{~m}$ of the 20 -m long working section was needed for flow development, after which mean flow and turbulence quantities were deemed constant or varying sufficiently slowly with streamwise distance to represent a spatially-homogenous boundary layer. This was longer than for the neutral baseline case, which was previously taken to be $10 \mathrm{~m}$. The second is in regard to the working-section inlet mean temperature profile. That is, i) starting with a uniform profile (case U1) and using the downstream measured profile to provide a (new) non-uniform inlet profile shape gave case $U 2$, ii) using the downstream profiles of this case to give a new inlet profile led to a another new profile in the downstream flow, case U3, which was only slightly different in shape from that of case U2. Repeating this process again would have led to an almost identical profile shape in the downstream flow. Thus, a natural equilibrium was established in which the inlet and downstream profiles had become the same in shape ${ }^{4}$. The layer is deeper by a factor of about 1.3 compared with that of the uniform inlet profile (i.e. $h$ for U3 compared with that for U1), though any tendency for more rapid streamwise development to nearly constant profiles in the downstream flow looks to be offset by the larger size of the structures. Nevertheless, when setting up a simulated unstable atmospheric boundary layer, this increase in height could be taken into account. For a given set of upstream flow generators and surface roughness distribution, two preliminary cycles appear to be sufficient in order to establish a working section inlet mean temperature profile.

\footnotetext{
${ }^{4}$ With a slightly higher level of temperature in the downstream flow because of no heat removal from the wind-tunnel roof.
} 
The cases U3 and U5, where the working section inlet temperature profile has been obtained iteratively from profiles measured in the downstream flow, agree well with those of Ohya and Uchida (2004) in a flow in which no artificial thickening was employed (other than a turbulence transition trip). It is interesting too that this concurrence has arisen where there is a significant difference in the stability imposed above. Allowing for differences in scale quantities (along the lines of Eq. 8) the imposed temperature gradient in their case was about four times larger than here.

Further conclusions have followed from comparing the measurements with established 'standard forms' for profiles of mean and turbulence quantities. Mean velocity and mean temperature profiles agreed well in the surface layer. Relationships for the Reynolds direct stresses agreed well in the surface layer, but not for the mean square of the temperature fluctuation. In the mixed layer, the converse was found: the Reynolds direct stresses were substantially larger in the experiments, but the mean square of the temperature fluctuation agreed well. By requiring the Reynolds stresses and the mean square temperature in the mixed layer to match those in the surface layer modified forms have been obtained. The modifications in effect amount to interpolations between the strongly unstable cases of the field and also laboratory measurements (e.g. Willis and Deardorff, 1974) used to obtain the standard forms, and the weakly unstable case of the present measurements, for which $u_{*}$ must remain a relevant scale.

The results partly conflict with the scaling laws of the mixed layer in that $w_{*}$ does not appear to be the only relevant scale for the velocity field, but the fluctuating temperature does scale upon $\tilde{\theta}_{*}$, even though this is defined in terms of $w_{*}$. Or, rather, equations such as Eq. 17 are not sufficiently general. It appears that as the surface heat flux increases the flow in the mixed layer becomes limiting, represented by the factor of 1.8 in Eq. 17. 


\section{Acknowledgements}

The work reported here was done under the SUPERGEN programme of the Engineering and Physical Sciences Research Council, SUPERGEN-Wind Phase 2, reference EP/H018662/1. Further details can be found from www.supergenwind.org.uk. The authors are particularly grateful to Prof. A. G. Robins, for useful discussions and comment. The EnFlo wind tunnel is a NERC/NCAS national facility, and the authors are also grateful to NCAS for the support provided.

\section{References}

Adrian RJ, Ferreira RTDS, Boberg T (1986) Turbulent thermal convection in wide horizontal fluid layers. Expts in Fluids 4: 121-141.

Armit, J, Counihan, J. (1968) The simulation of the atmospheric boundary layer in a wind tunnel. Atmos Environ 2: 49-71.

Beljaars ACM, Holtslag AAM (1991) Flux parameterization over land surfaces for atmospheric models. J Appl. Meteorol 30: 327-341.

Caughey SJ, Palmer SG (1979) Some aspects of turbulence structure through the depth of the convective boundary layer. Q J R Meteorol Soc 105: 811-827.

Caughey SJ, Wyngaard (1979) The turbulence energy budget in convective conditions. Q J R Meteorol Soc 105: 231-239.

Counihan, J. (1969) An improved method of stimulating atmospheric boundary layers in a wind tunnel. Atmos Environ 3: 197-214. 
ESDU (2001) Characteristics of atmospheric turbulence near the ground. Part II: Single point data for strong winds (neutral atmosphere). ESDU 85020.

Engineering Sciences Data Unit., London, UK, 42 pp.

ESDU (2002) Strong winds in the atmospheric boundary layer. Part 1: hourlymean wind speeds. ESDU 85026. Engineering Sciences Data Unit., London, $\mathrm{UK}, 61 \mathrm{pp}$.

Gryning SE, Holtslag AAM, Irwin JS, Sivertsen B. (1987) Applied dispersion modelling based on meteorological scaling parameters. Atmos Environ 21: 78-89.

Fedorovich E, Neiuwstadt FTM, Kaiser, R. (2001) Numerical and laboratory study of a horizontally evolving convective boundary layer. Part 1: Transition regimes and development of the mixed layer. J Atmos Sci 58: 70-86.

Hancock, PE, Bradshaw, P. (1989) Turbulence structure of a boundary layer beneath a turbulent free stream. J Fluid Mech 205: 45-76.

Hancock PE, Pascheke F (2013) Wind tunnel simulation of the wake flow of a large wind turbine in a stable boundary layer: Part 1, the boundary layer simulation. EnFlo Internal Report, FRC/TG/13.33

Hancock PE, Zhang S, Hayden (2012) A wind-tunnel artificially-thickened weakly-unstable atmospheric boundary layer. EnFlo Internal Report, $\mathrm{FRC} / \mathrm{TG} / 13.31$

Holtslag AAM, Nieuwstadt FTM (1986) Scaling the atmospheric boundary layer. Boundary-Layer Meteorol 36: 201-209.

Holtslag AAM, Meong, CH (1991) Eddy diffusivity and countergradient transport in the convective atmospheric boundary layer. J Atmos Sci 48: 16901698. 
Hunt JCR, Kaimal JC, Gaynor, JE (1988) Eddy structure in the convective boundary layer - new measurements and new concepts. Q J R Meteorol Soc 114: $827-858$.

Huynh BP, Coulman CE, Turner TR (1990) Some turbulence characteristics of convectively mixed layers over rugged and homogeneous terrain.

Boundary-Layer Meteorol 51: 229-254.

Irwin HPAH (1981) The design of spires for wind stimulation, J Wind Eng and Ind Aerodyn 7: 361-366.

Kaimal JC, Wyngaard JC, Haugen DA, Coté OR, Izumi, Y. (1976) Structure in the convective boundary layer. J Atmos Sci 33: 2152-2169.

Kaimal JC, Finnigan JJ (1994) Atmospheric boundary layer flows; their structure and measurement. Oxford University Press, UK, 302 pp.

Lenschow DH, Wyngaard JC, Pennell WT (1980) Mean-field and second-moment budgets in a baroclinic, convective boundary layer. J Atmos Sci 37: 1313-1326.

Magnusson M, Smedman A-S (1996) A practical method to estimate the wind turbine wake characteristics from turbine data and routine wind measurements. J Wind Eng 20: 73-92.

Nieuwstadt FTM, van Dop H (1982) Atmospheric turbulence and air pollution modelling. D Reidel Publishing Company. NL, 379 pp.

Ohya Y, Uchida, T (2004) Laboratory and numerical studies of the convective boundary layer capped by a strong inversion. Boundary-Layer Meteorol 112: 223240 .

Robins A G, Castro I P, Hayden P, Steggel N, Contini D, Heist D and Taylor T J (2001) A wind tunnel study of dense gas dispersion in a stable boundary layer over a rough surface. Atmos Environ 35: 2253-2264. 
Stull RB (1988) An introduction to boundary layer meteorology.

Kluwer Academic Publishers, NL, 679 pp.

Willis GE, Deardorff JW (1974) A laboratory model of the unstable planetary boundary layer. J Atmos Sci 31: 1297-1307.

Wyngaard JC (1992) Atmospheric turbulence. Ann Rev Fluid Mech 24: 205235 .
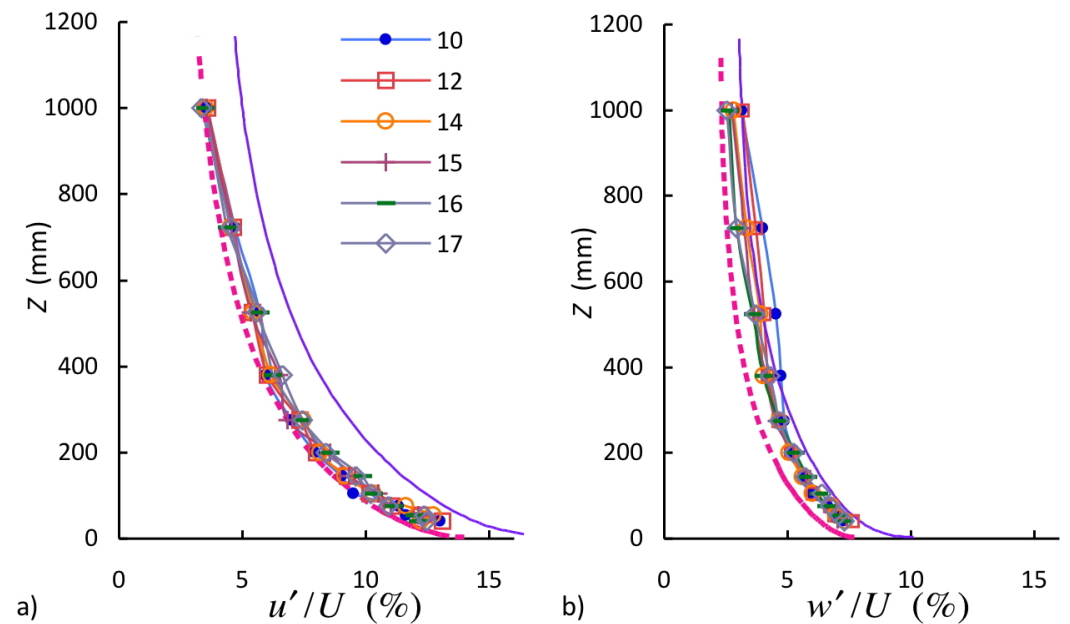

Fig. $1 u^{\prime} / U$ and $w^{\prime} / U$ in the neutral boundary layer from $X=10 \mathrm{~m}$ to $17 \mathrm{~m}$, compared with the ESDU guidelines.

Full and broken lines are for $z_{0}$ (full scale): full, $0.005 \mathrm{~m}$; broken, $0.0005 \mathrm{~m}$. 

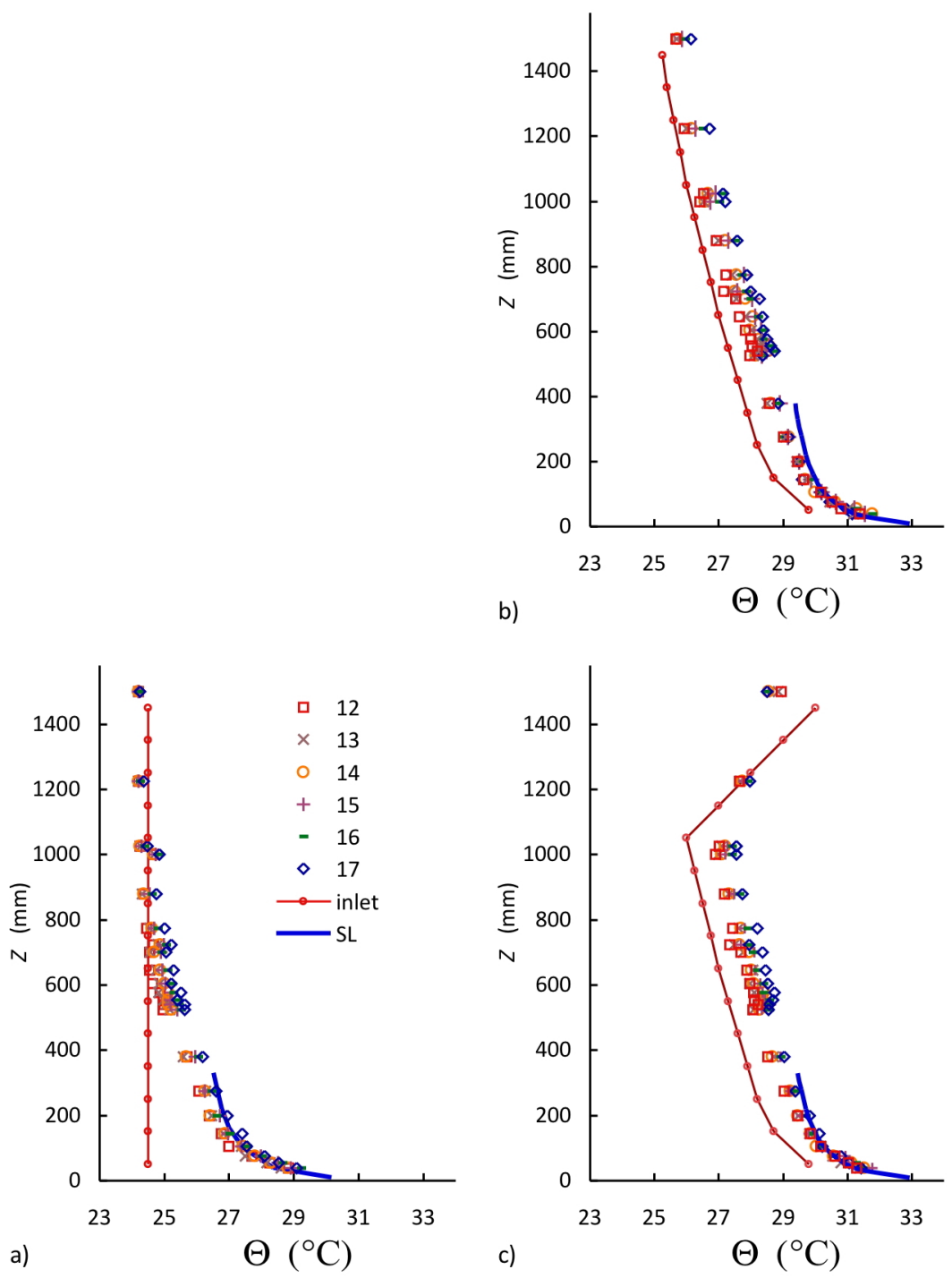

Fig. 2 Mean temperature profiles, a) U1; b) U3; c) U5

Symbols for b) and c) as in a), for profiles between $X=12 \mathrm{~m}$ and $17 \mathrm{~m}$.

Full line with symbol is the inlet profile. Full line no symbol is surface layer, Eq. 12. Streamwise distance, $X$, as in later figures, is shown in units of $\mathrm{m}$. 

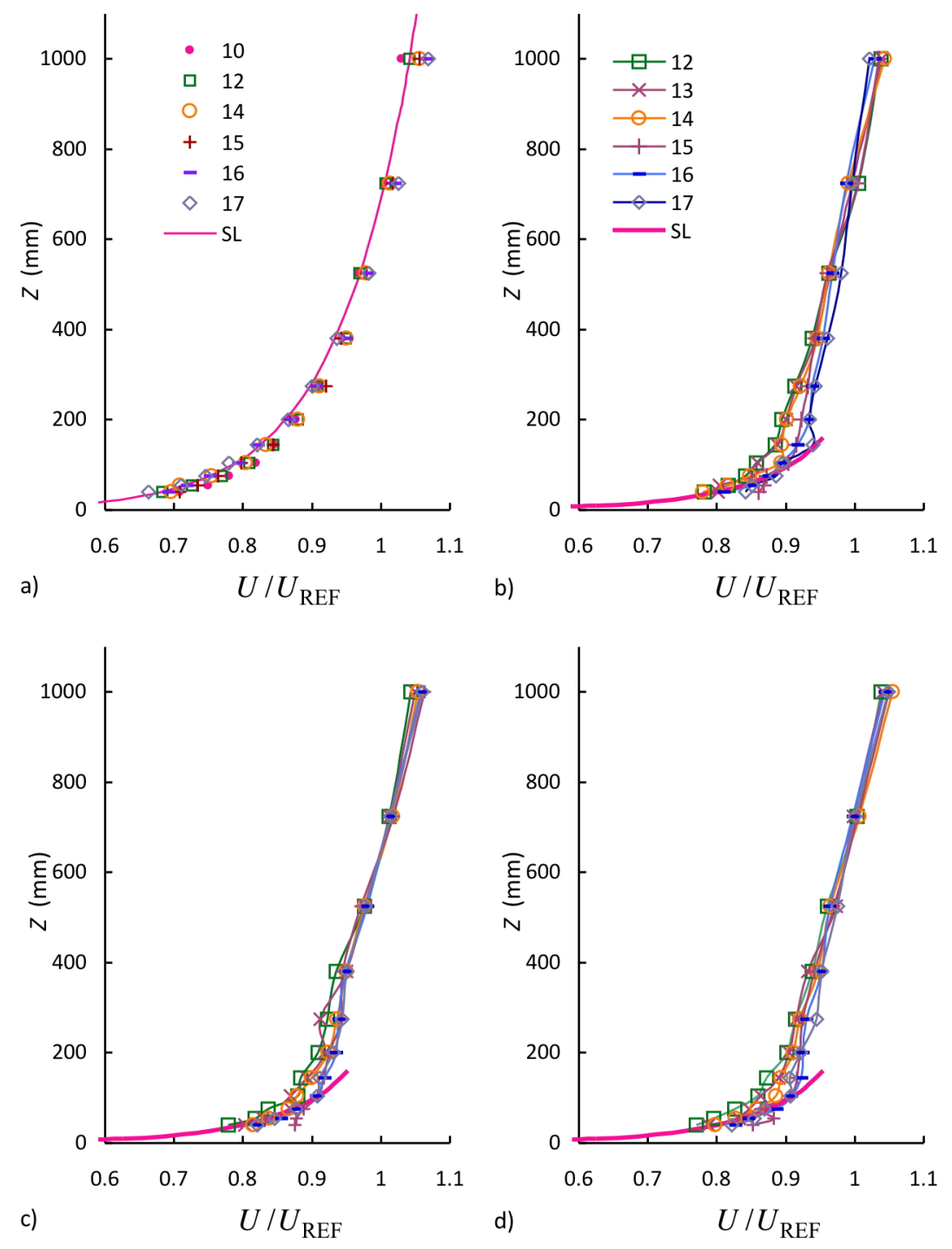

Fig. 3 Mean velocity profiles, a) N; b) U1; c) U3; d) U5

Symbols for c) and d) as in b). Solid line is surface layer, Eq. 11 . 

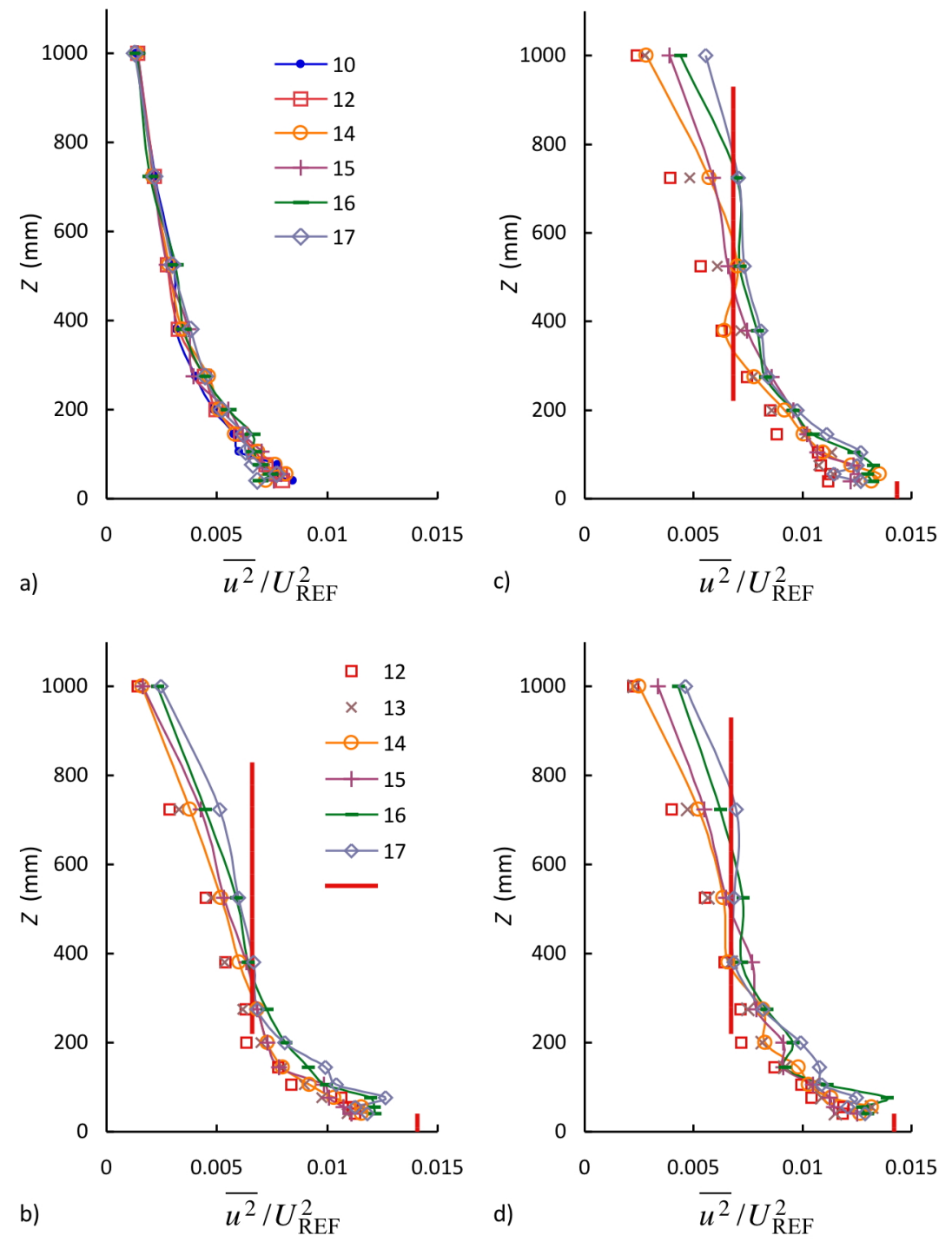

Fig. 4 Reynolds direct stress $\overline{u^{2}} / U_{\text {ref }}^{2}$ : a) N; b) U1; c) U3; d) U5 Lines, Eqs 13 and 26. Symbols for c) and d) as in b). 

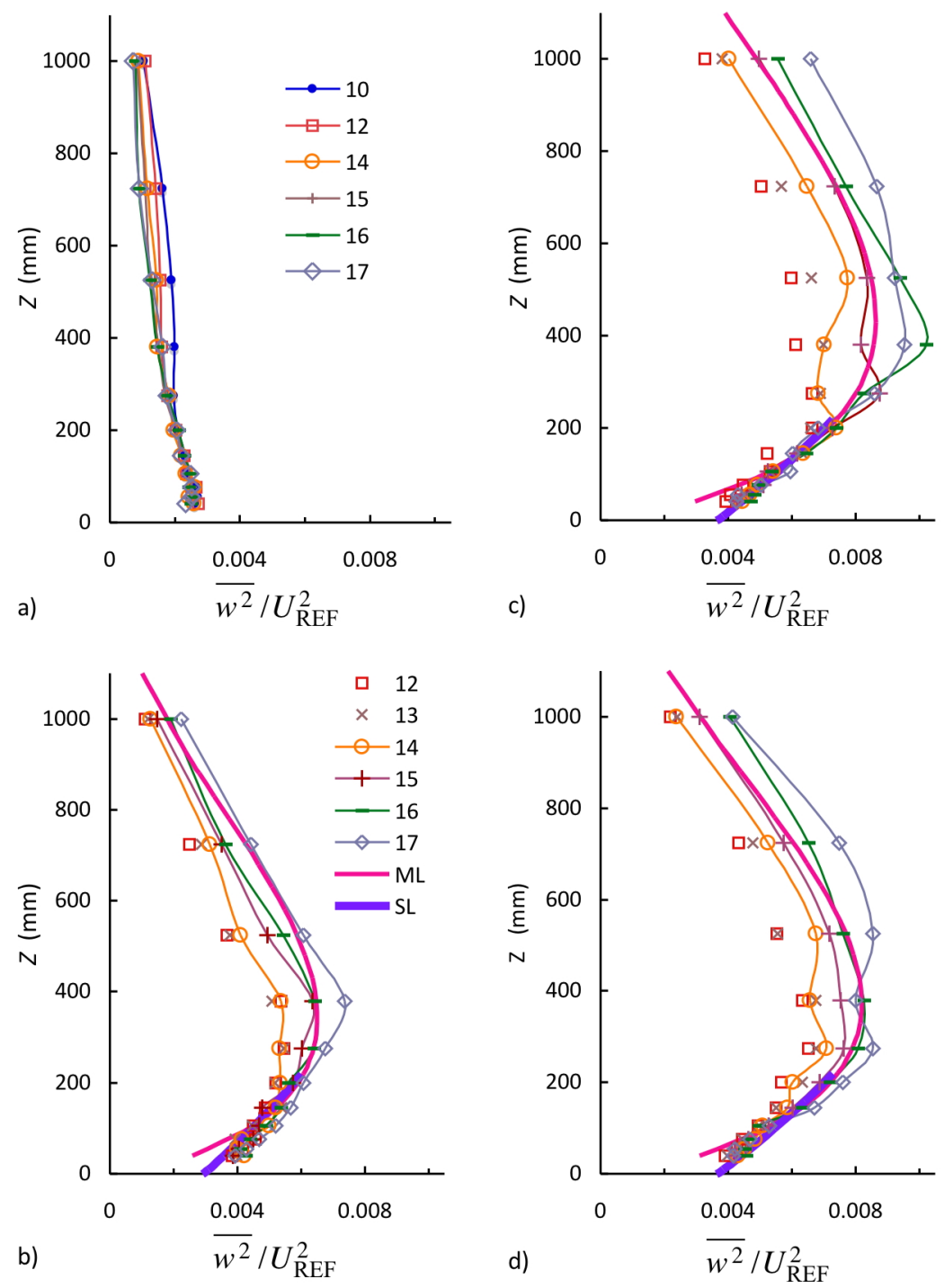

Fig. $5 \quad$ Reynolds direct stress $\overline{w^{2}} / U_{\text {ref }}^{2}$ : a) N; b) U1; c) U3; d) U5 Lines, Eqs 23 and 24. Symbols for c) and d) as in b). 

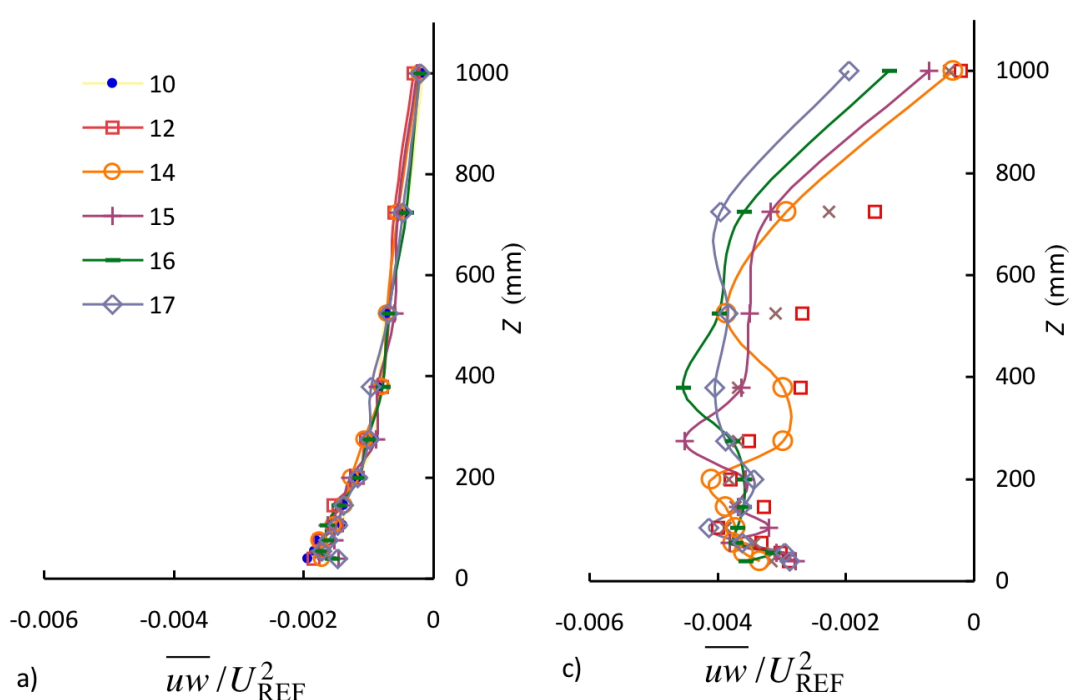

a) $\quad \overline{u w} / U_{\mathrm{REF}}^{2}$

c) $\overline{u w} / U_{\mathrm{REF}}^{2}$

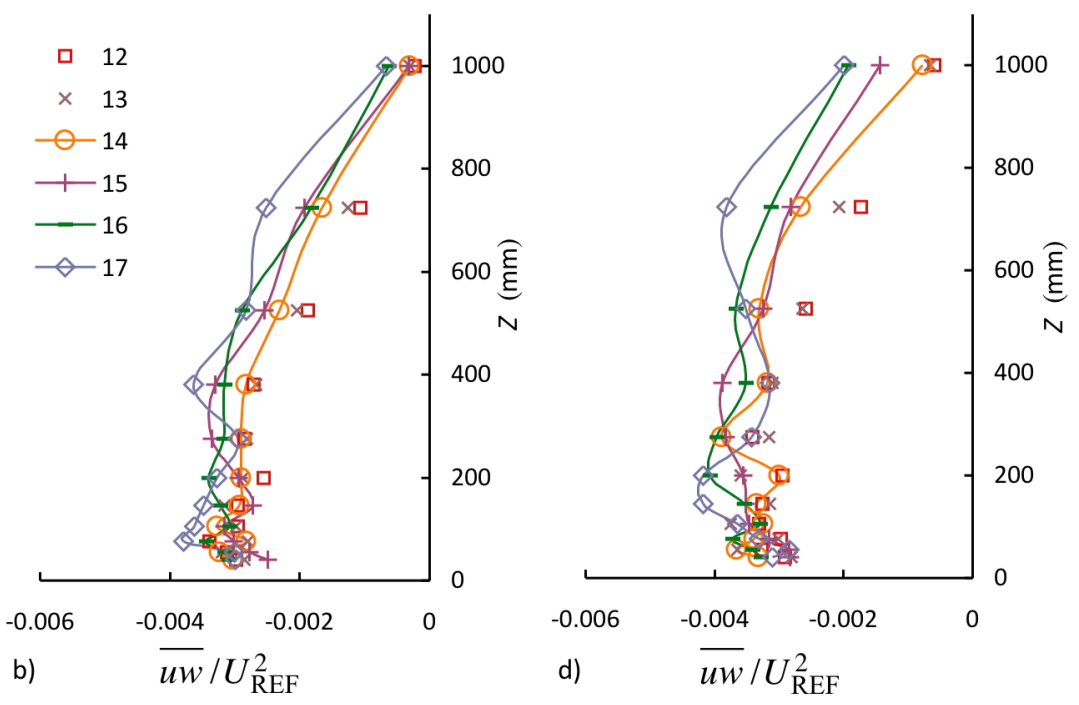

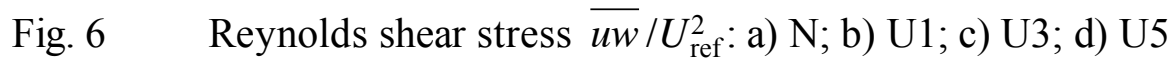

Symbols for c) and d) as in b). 

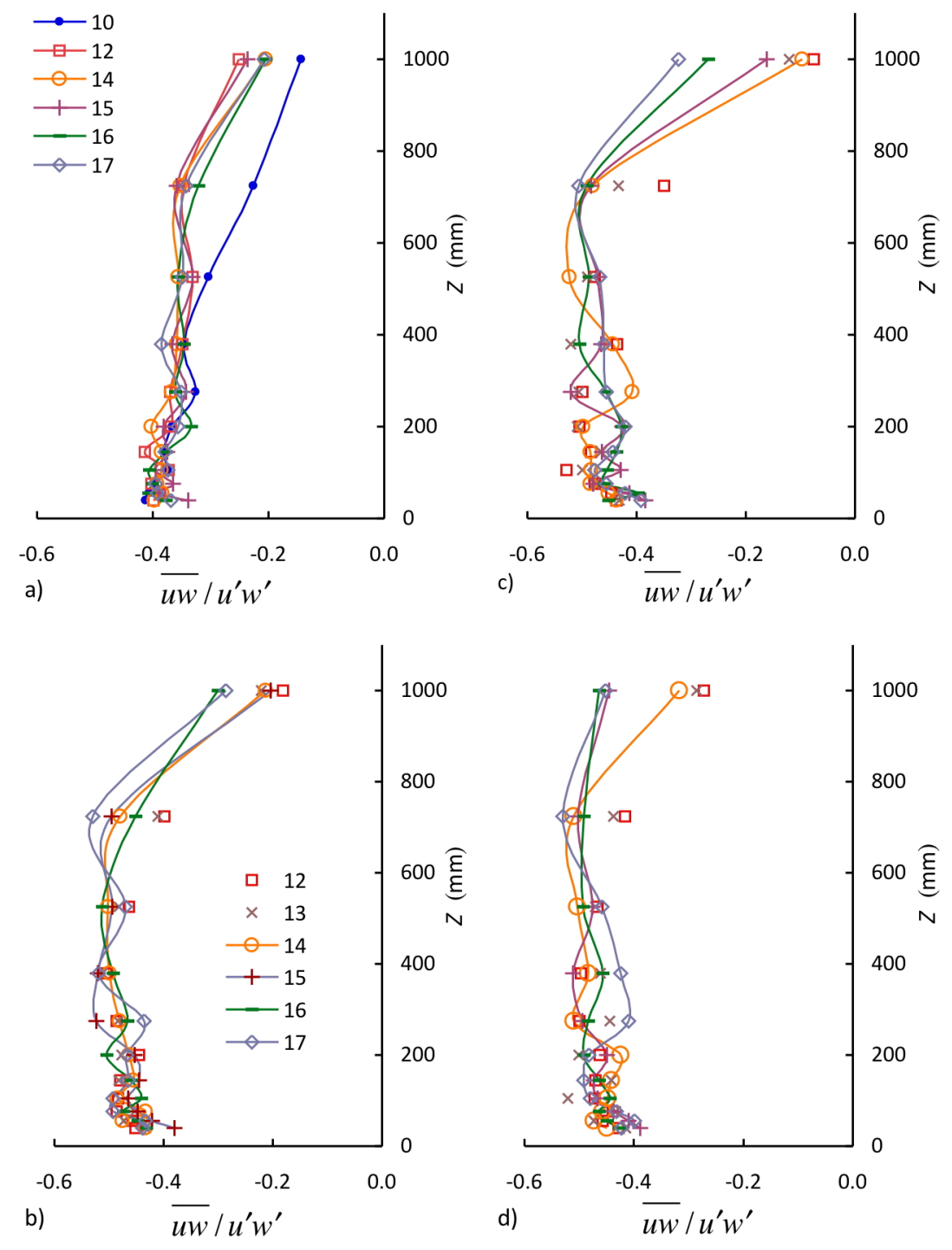

Fig. 7 Correlation coefficient $\overline{u w} / u^{\prime} w^{\prime}:$ a) N; b) U1; c) U3; d) U5 Symbols for c) and d) as in b). 

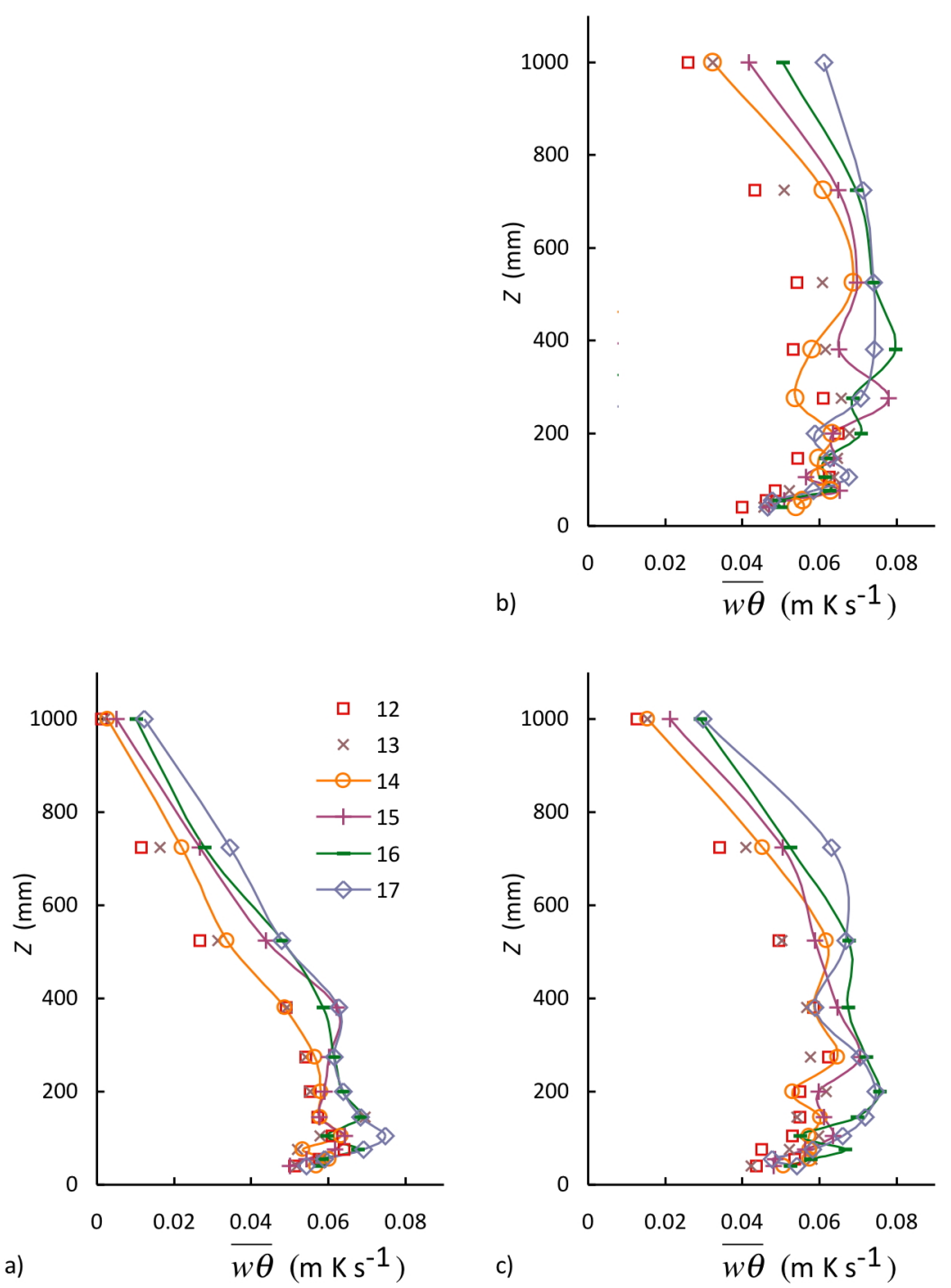

Fig. $8 \quad$ Kinematic heat transfer, $\overline{w \theta}$ : a) U1; b) U3; c) U5 Symbols for b) and c) as in a). 

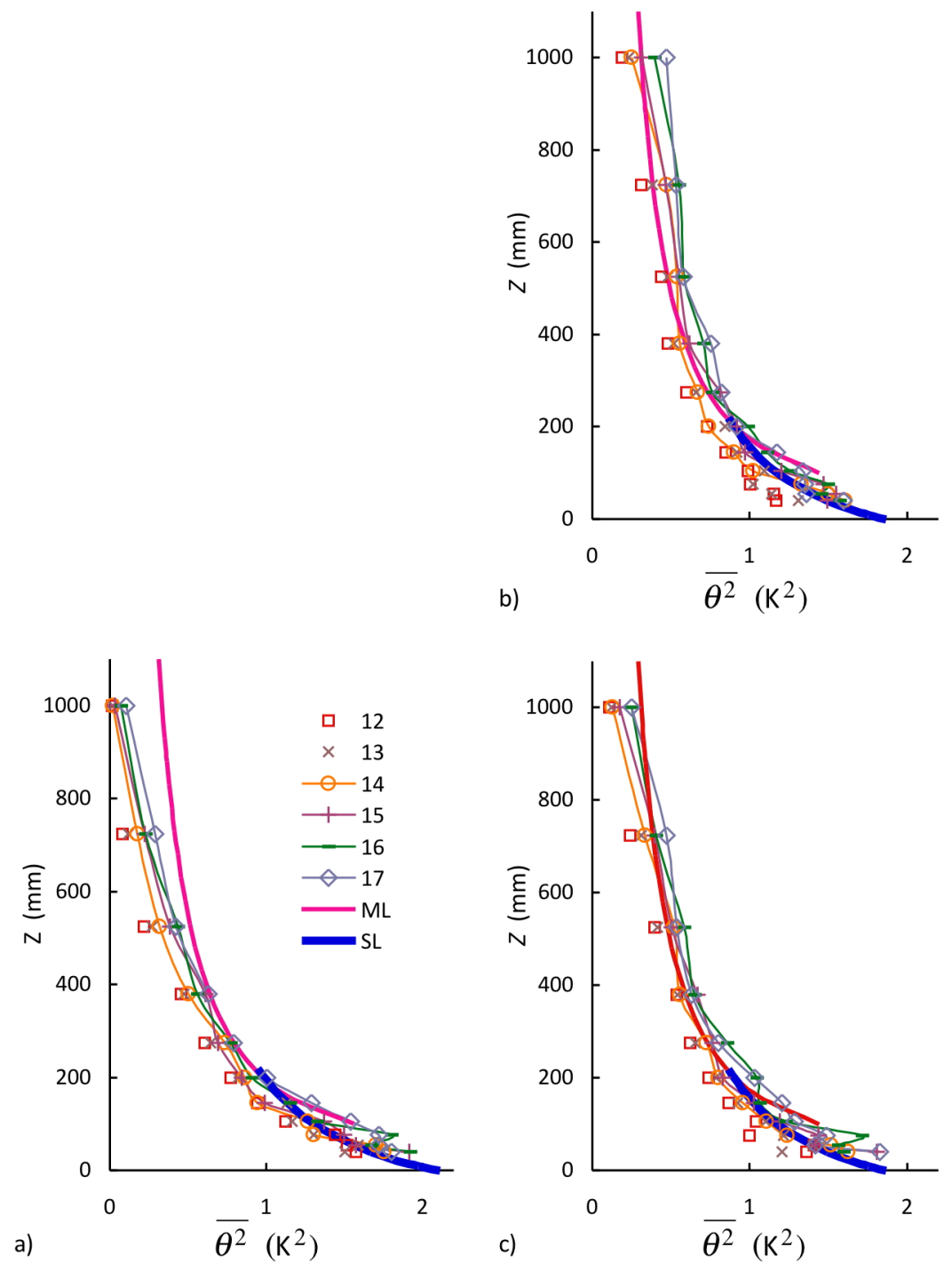

Fig. 9 Mean square temperature fluctuation, $\overline{\theta^{2}}$ : a) U1; b) U3; c) U5 Lines: Eqs 18 and 19 

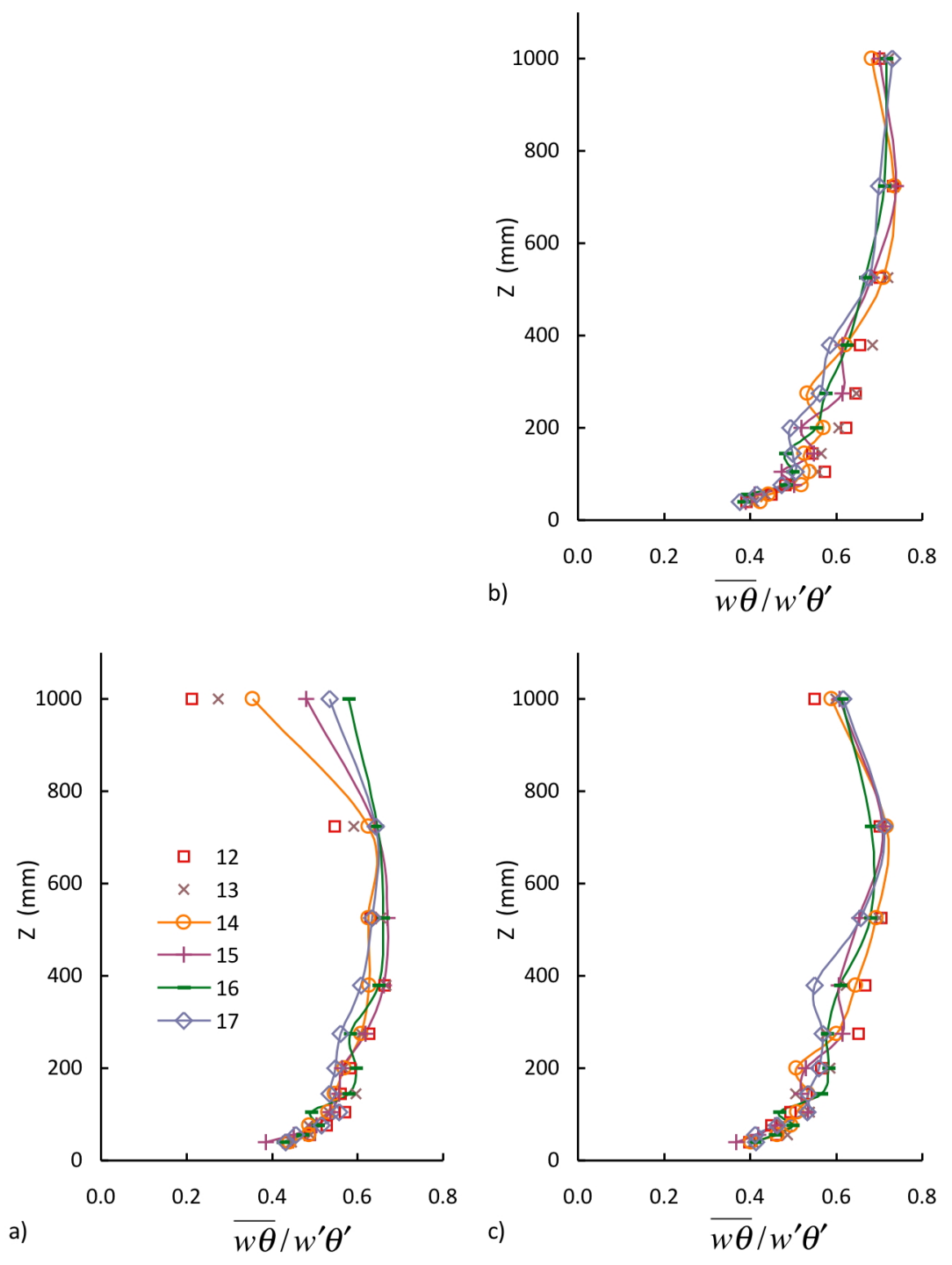

Fig. 10 Correlation coefficient $\overline{w \theta} / w^{\prime} \theta^{\prime}$ : a) U1; b) U3; c) U5 Symbols for b) and c) as in a). 

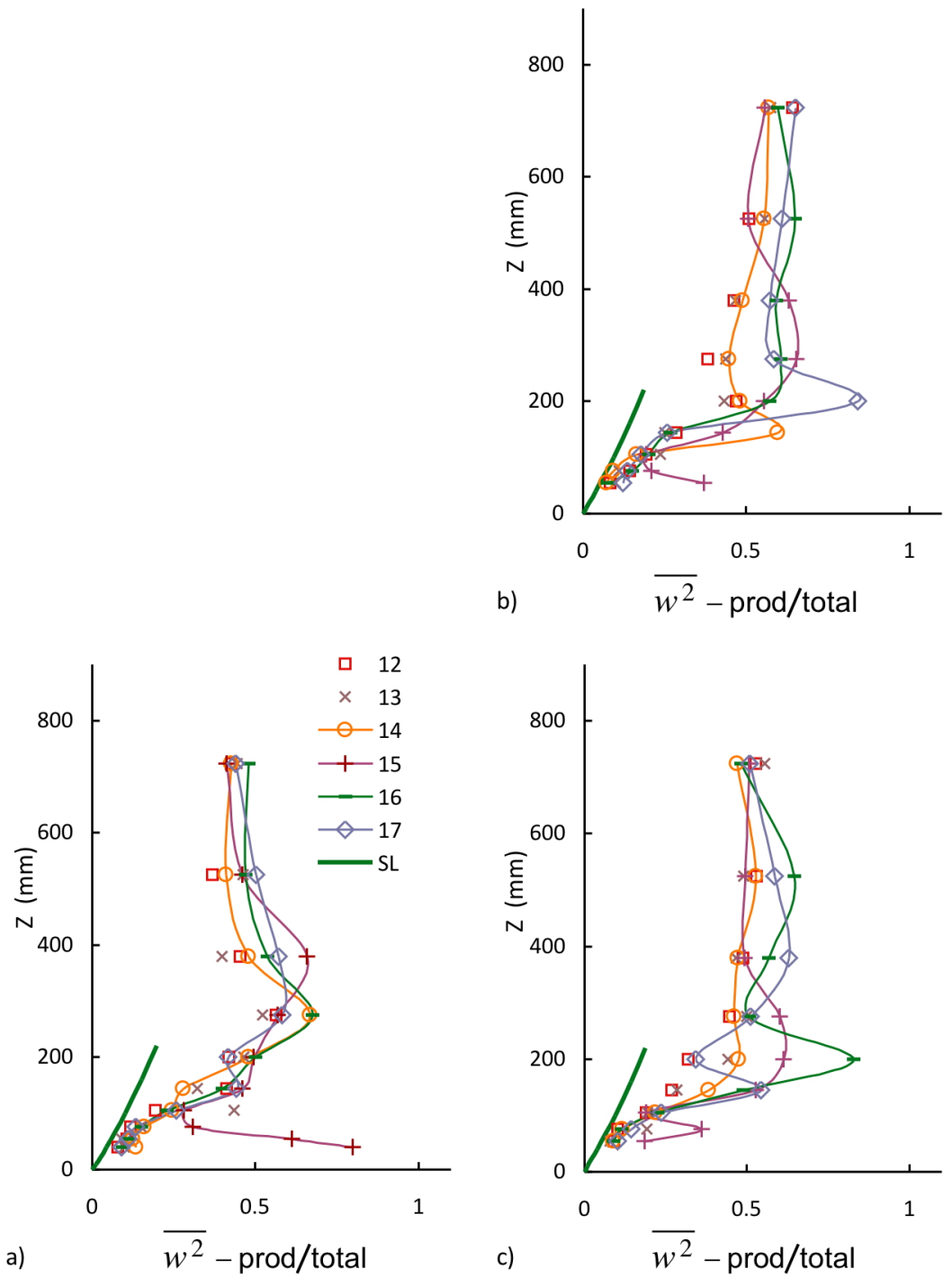

Fig. 11 Buoyant production as a fraction of the total production of turbulent kinetic energy: a) U1; b) U3; c) U5

Symbols for b) and c) as in a). Line shows ratio for surface-layer scaling. 

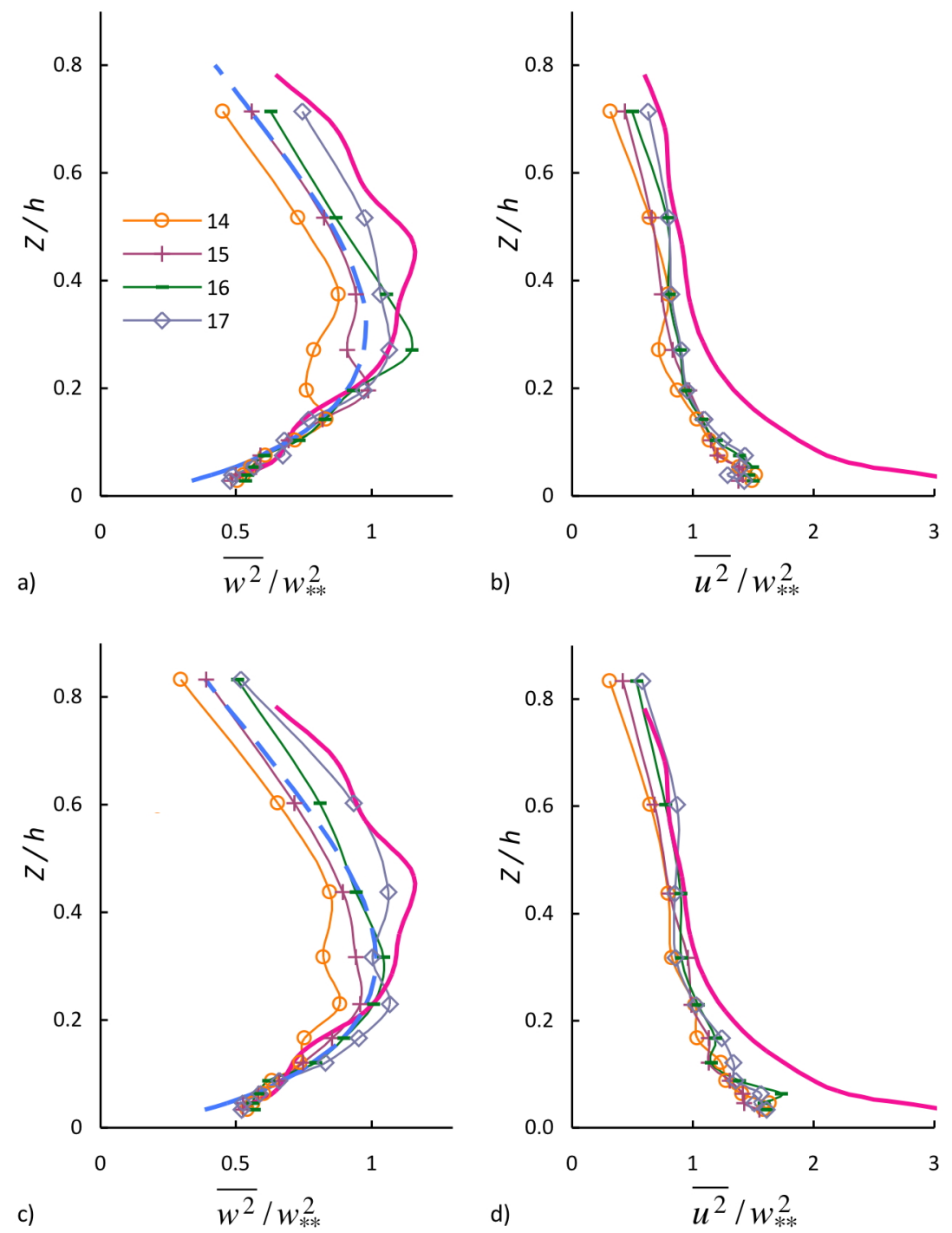

Fig. 12 Profiles of $\overline{w^{2}}$ and $\overline{u^{2}}$ compared with the measurements of Ohya and Uchida (2004): a) and b) U3; c) and d) U5

Symbols as in a). Full line: Ohya and Uchida (2004). Broken line: Eq. 24. 

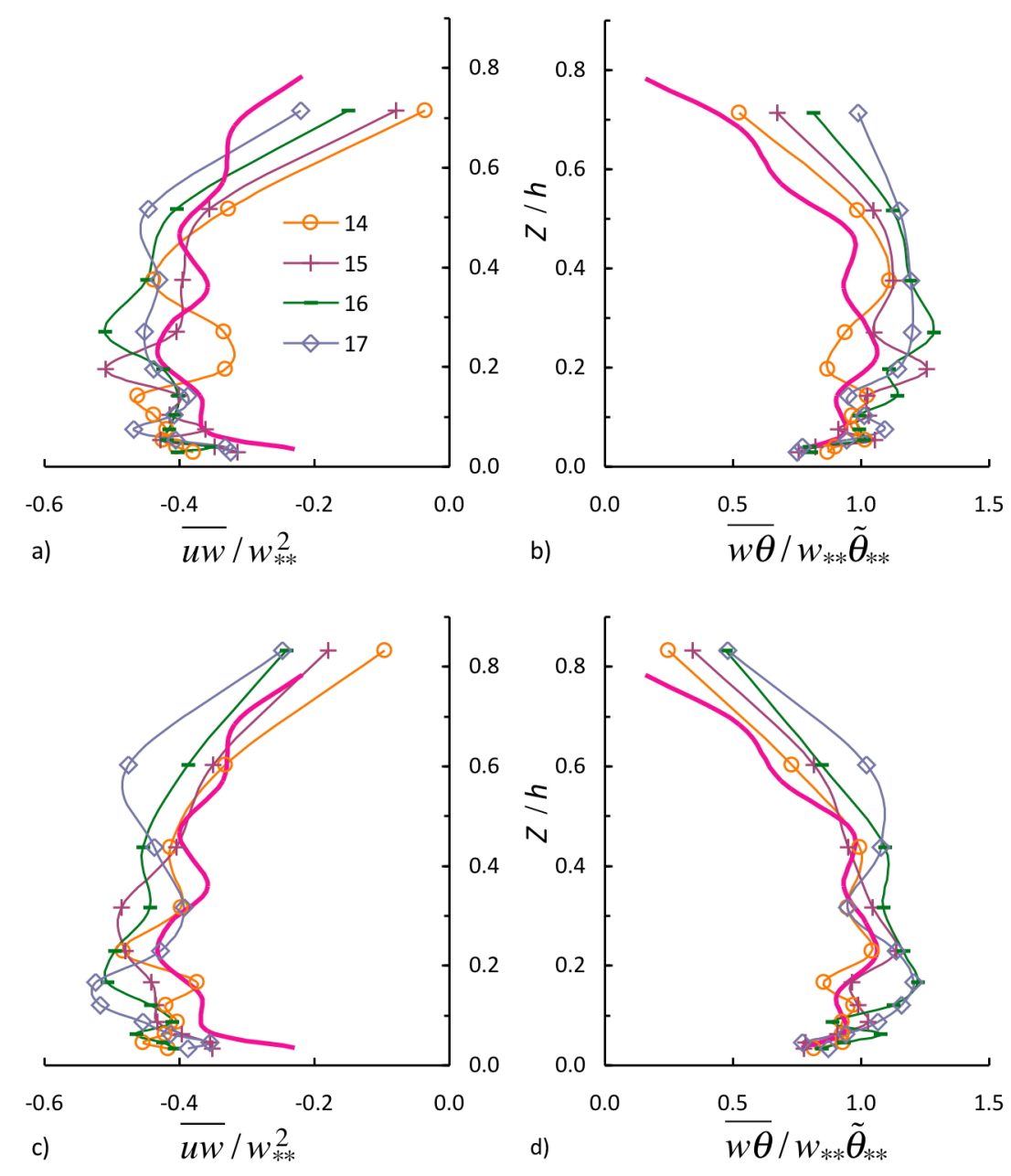

Fig. 13 Profiles of $\overline{u w}$ and $\overline{w \theta}$ compared with the measurements of Ohya and Uchida (2004): a) and b) U3; c) and d) U5

Symbols as in a). Full line: Ohya and Uchida (2004). 